\title{
Credit scoring development in the light of the new Brazilian General Data Protection Law
}

\author{
Robinson A. A. de Oliveira Junior \\ Universidade de São Paulo, Brazil \\ robinson@usp.br
}

\begin{abstract}
With the advent of the new Brazilian General Data Protection Law (LGPD) which determines the right to the explanation of automated decisions, the use of non-interpretable models for human beings, known as black boxes, for the purposes of credit risk assessment may remain unfeasible. Thus, three different methods commonly applied to credit scoring - logistic regression, decision tree, and support vector machine (SVM) - were adjusted to an anonymized sample of a consumer credit portfolio from a credit union. Their results were compared and the adequacy of the explanation achieved for each classifier was assessed. Particularly for the SVM, which generated a black box model, a local interpretation method - the SHapley Additive exPlanation (SHAP) - was incorporated, enabling this machine learning classifier to fulfill the requirements imposed by the new LGPD, in equivalence to the inherent comprehensibility of the white box models.
\end{abstract}

CCS Concepts: • Computing methodologies $\rightarrow$ Supervised learning by classification.

Keywords: Classification models, Credit scoring, Interpretability, LGPD, Machine learning

\section{INTRODUÇÃO}

Modelos de credit scoring foram uma das primeiras ferramentas de gestão de risco desenvolvidas e também uma das primeiras a admitir dados comportamentais [Thomas et al. 2002]. Vitais à expansão do crédito ao consumidor ao longo das últimas décadas, sua relevância é incontestável, bem como a importância e o protagonismo presentes da inteligência artificial.

Entretanto, os meios utilizados para se predizer um evento que possa impactar vidas humanas de forma tão profunda, a exemplo da atribuição de um perfil capaz de regular o acesso individual a bens, serviços ou recursos financeiros, não são incontestes. E a Lei Geral de Proteção de Dados (LGPD), Lei $\mathrm{n}^{\mathrm{O}}$ 13.709/18 ${ }^{1}$, inspirada no Regulamento Geral sobre a Proteção de Dados (General Data Protection Regulation (GDPR)) da União Europeia [Erickson 2019], assegura aos titulares dos dados direitos de informação sobre a lógica de processos de tratamento de dados e de revisão de decisões tomadas unicamente com base no tratamento automatizado de dados pessoais que afetem seus interesses.

Para modelos de credit scoring baseados em técnicas estatísticas, a exemplo da análise de discriminantes lineares e da regressão logística, a predição pode ser interpretada a partir de seus coeficientes [Hand and Henley 1997]. Em contraste, para alguns modelos baseados em técnicas de aprendizado de máquina específicas, como redes neurais artificiais, máquinas de vetores de suporte e redes neurais profundas, não é possível identificar como são determinadas as suas predições, sendo frequentemente denominados caixas-pretas [Molnar 2020], algo incompatível com a LGPD.

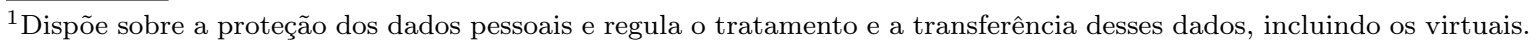

Copyright(C)2021. Permission to copy without fee all or part of the material printed in KDMiLe is granted provided that the copies are not made or distributed for commercial advantage, and that notice is given that copying is by permission of the Sociedade Brasileira de Computação. 
Dentre as técnicas mais recentes para se incorporar interpretabilidade aos modelos caixas-pretas, independentemente do modelo preditivo previamente induzido (model-agnostic), há a SHapley Additive exPlanation (SHAP) ${ }^{2}$, uma técnica estado da arte, fundamentada na teoria dos jogos cooperativos, capaz de determinar a contribuição marginal média ponderada de um atributo qualquer ao valor de saída do modelo [Molnar 2020].

Diante do exposto, este artigo, que é uma síntese de uma pesquisa [Oliveira-Junior 2021], objetiva aplicar sobre uma amostra anonimizada de uma carteira de crédito ao consumidor de uma cooperativa de crédito, alguns dos métodos de classificação aplicados para credit scoring mais difundidos ${ }^{3}-(\mathrm{i})$ um probabilístico, de regressão logística, (ii) um baseado em procura, de árvore de decisão, e (iii) um baseado em otimização, de máquina de vetores de suporte ${ }^{4}$, com especial interesse pelo último, por se tratar de um método caixa-preta - comparando os resultados obtidos e avaliando a adequabilidade da explicação obtida para cada classificador, seja ela aparente ou alcançada a partir do emprego do método de interpretação local, à luz da nova LGPD.

No Brasil, publicações acerca da interpretabilidade são relativamente recentes, destacando-se a dissertação "A semiotic view on the interpretability of machine learning models" [Santo 2018], em que o autor sugere uma sistemática para lidar com o desafio da interpretabilidade. Em 2020, segundo Oliveira-Junior (2021), a busca conjunta pelas palavras-chave associadas à pesquisa na plataforma "Google Acadêmico" " não retornou resultados. Em virtude da maior compatibilidade com o tema proposto e área de aplicação, pode ainda ser citada a dissertação "A utilização de algoritmos de aprendizado de máquina em problemas de classificação" [Batista 2018].

Assim, este artigo visa contribuir com os estudos sobre interpretabilidade ao apresentar o uso da SHAP incorporado ao processo de modelagem de credit scoring a fim de permitir a utilização de modelos caixas-pretas no processo de formação de perfis de crédito, em conformidade com os requisitos da nova LGPD.

\section{METODOLOGIA}

Foi extraída uma amostra com 7.761 decisões de crédito (observações) ocorridas entre os anos de 2016 e 2018, e apoiada em 59 atributos - relacionados a dados cadastrais (13) e comportamentais dos cooperados (11), a dados das operações (32) e de performance (3) - oriunda de uma cooperativa de crédito e limitada ao público pessoa física. O conceito de mau (mau pagador) empregado foi de 60 dias de atraso em uma janela de 12 meses, de modo a tornar exequível a classificação ${ }^{6}$.

Para a análise exploratória, realizada em Python, foram verificadas a estrutura do conjunto de dados, as medidas básicas de tendência central e de dispersão, o tipo dos atributos e outras características, tais como sua não-nulidade, valores faltantes, cardinalidade e eventuais redundâncias. Tendo em vista o conceito de mau escolhido, as 7.761 observações iniciais se dividiam em 7.663 bons pagadores, dita classe negativa ("0"), e 98 maus pagadores, dita classe positiva ("1").

Uma parcela dos atributos originais permitiu a geração de novos atributos (40), os quais, em sua maioria, correspondiam a sumarização de informações: por exemplo, o valor mensal para percentual

\footnotetext{
${ }^{2}$ Embora a Local Interpretable Model-agnostic Explanations (LIME) seja um método de interpretação local pioneiro, que induz um modelo substituto capaz de criar uma aproximação à predição que se deseja explicar a partir de propriedades da instância em questão [Ribeiro et al. 2016], possibilitando que os coeficientes deste sejam utilizados para se obter a explicação pretendida, a técnica foi preterida por não oferecer precisão equivalente a SHAP [Molnar 2020].

${ }^{3} \mathrm{~A}$ seleção dos métodos teve como base uma revisão sistemática de métodos de classificação aplicados para credit scoring [Louzada et al. 2016]: https://doi.org/10.1016/j.sorms.2016.10.001

${ }^{4} \mathrm{O}$ método de redes neurais artificiais foi preterido em detrimento da máquina de vetores de suporte, pois se relatou observar com mais frequência que o primeiro perdia desempenho preditivo diante de um conjunto de dados desbalanceado [Louzada et al. 2016].

${ }^{5}$ https://scholar.google.com.br/?hl=pt

${ }^{6}$ Dado o envolvimento do cooperado com a cooperativa, atrasos superiores a 90 dias são pouco usuais.
} 
de utilização do cheque especial produziu quatro novos atributos, apresentando a média de utilização nos (i) 3, (ii) 6 e (iii) 12 meses que antecederam a decisão de crédito, além do (iv) percentual máximo de utilização no período.

Após visualização das distribuições, histogramas, boxplots e contagens - também observadas sob a perspectiva de cada classe à variável resposta, e a partir dos dados normalizados - agrupamento de categorias, discretização de atributos, identificação e tratamento de outliers, exame da correlação linear entre as variáveis independentes (previsoras) e a variável dependente (resposta), e estimação do Weight of Evidence (WoE) ${ }^{7}$ e Information Value (IV) ${ }^{8}$, foram descartados 76 atributos, restando apenas 23 - 11 originais e 12 novos - mais a variável resposta ("TARGET"), conforme tabela I a seguir, sendo preservadas 5.149 observações, com 5.062 bons pagadores e 87 maus pagadores.

\begin{tabular}{|c|c|c|c|c|}
\hline IDADE & ESTADOCIVIL & NUMERODEPENDENTES & ESCOLARIDADE & RENDA \\
\hline MUNICIPIO & PA & PRODUTO & SUBREGIAO \\
\hline IAP_DISC & CC_ATIVO_FLAG & LIQUIDADOS_DISC & REPACTUOU_FLAG & DEV_ALINEAS_11E12_FLAG \\
\hline SALDO_MD_12M & PERC_MD_CHQ_ESP_12M & PERC_MAX_CHQ_ESP & NRO_MAX_DIAS_AD & NRO_MAX_MESES_AD \\
\hline SALDO_MD_VENCIDOS_12M & SALDO_MAX_VENCIDOS & NRO_MAX_MESES_ACIMA_60 & TARGET & \\
\hline
\end{tabular}

Tabela I. Atributos restantes.

Verde: atributos originais; Amarelo: atributos novos.

Fonte: Elaborada pelo autor.

A análise exploratória no conjunto de dados obtido apresentou uma realidade bastante desafiadora para a modelagem de credit scoring. Dentre as razões, podem ser destacadas:

- ao solicitar que não houvesse repetições em diferentes pontos de observação, substituindo solicitantes "repetidos" por "novos", o volume médio de decisões de crédito mensais foi de apenas 216 (7.761 $36)$;

-baixíssima frequência à classe positiva (maus pagadores), culminando em um conjunto de dados extremamente desbalanceado (ao final, apenas $1,69 \%$ dos exemplos $-87 \div 5.149$ );

- embora as operações rodem em sistemas transacionais, inúmeras características de grande relevância à modelagem de credit scoring não eram registradas à ocasião da tomada de decisão - ex. dados de birôs e do Sistema de Informações de Créditos (SCR) do Banco Central do Brasil;

-uma correlação linear insignificante entre os atributos preditores e a variável resposta.

\section{DESENVOLVIMENTO DA MODELAGEM DE CREDIT SCORING}

O conjunto de dados foi separado em treino e teste usando a função "train_test_split" do pacote "sklearn", com $20 \%$ para o conjunto de teste, e mantendo a proporção observada às classes da variável resposta no conjunto de dados.

Aos atributos numéricos foi aplicado o "StandardScaler" para padronização dos dados, também do "sklearn", removendo a média e reescalando a variância para uma unidade. Os atributos categóricos foram transformados usando a classe referente ao Weight of Evidence do pacote "Category Encoders".

Antes de lidar com o desbalanceamento do conjunto - é esperado que diferenças observadas nas frequências das classes afetem significativamente a previsibilidade dos modelos - foi estabelecido um classificador de referência ou baseline: uma árvore de decisão sobre a amostra desbalanceada utilizando grid search para otimização de hiperparâmetros, ambos do pacote "sklearn", e validação cruzada com 3 folds ${ }^{9}$, após divisão estratificada dos conjuntos de treino e teste.

\footnotetext{
${ }^{7}$ Aponta o poder preditivo de cada categoria de uma variável independente em relação à variável dependente.

${ }^{8}$ Valor único que aponta todo o poder preditivo de uma variável, bastante útil à seleção de atributos.

${ }^{9}$ Dividindo o conjunto de treinamento em $k$ subconjuntos de mesmo tamanho e mutuamente exclusivos, onde $k=3$, e utilizando então um subconjunto para teste e os $k-1$ restantes para estimação dos parâmetros.
} 
A acurácia obtida foi de 97,38\%, porém a medida não possui qualquer utilidade por força do desbalanceamento observado, tendo concentrado as predições à classe majoritária, ou seja, classificando as observações no conjunto de teste como bons pagadores. Para a decisão de crédito é importante que o modelo apresente alta revocação ${ }^{10}$, pois o custo de confundir um mau pagador com um bom pagador costuma ser elevado. Ao mesmo tempo, não se pode prescindir da precisão ${ }^{11}$, uma vez que classificar bons pagadores como maus pagadores pode restringir negócios, e o crédito é a maior fonte de receita das instituições financeiras.

Por sua vez, a medida $F$ corresponde à média harmônica da precisão e da revocação. Como não podemos desacolher ambas, nos caberia apenas ajustá-la a fim de redistribuir pesos, conferindo maior importância à revocação: tal medida é conhecida como $F_{2}$ e é expressa por $\left[\left(1+2^{2}\right) \times\right.$ precisão $\times$ revocação $] \div\left[2^{2} \times\right.$ (precisão + revocação) $]$. Para o classificador de referência, precisão, revocação e medida $F_{2}$ atingiram $18,75 \%, 17,65 \%$ e $17,86 \%$, respectivamente.

$\mathrm{Na}$ sequência foi desenvolvido um pipeline para implementar as técnicas de balanceamento ${ }^{12}$ - tão somente no conjunto de treino - e ajustar os modelos de regressão logística, de árvore de decisão e de máquina de vetores de suporte, disponíveis no pacote "sklearn", em conjunto com a utilização de grid search para otimização de hiperparâmetros e validação cruzada com 3 folds, concluindo sua execução com a exibição da matriz de confusão e medidas de avaliação ${ }^{13}$. Abaixo, na tabela II, são exibidos os melhores modelos obtidos para cada método, incluindo técnica de balanceamento e hiperparâmetros:

\begin{tabular}{|c|c|c|c|c|c|c|c|c|c|c|c|c|c|}
\hline Classificador & Técnica & Hiperparâmetros & $\mathrm{VN}$ & FN & $\mathrm{VP}$ & FP & Precisão & Revocação & $F_{2}$ & Acurácia & Acurácia Balanceada & Especificidade & ROC AUC \\
\hline LR & ROS & C: ' $100.0^{\prime}$ & 849 & 5 & 12 & 164 & 0.0682 & 0.7059 & 0.2459 & 0.8359 & 0.772 & 0.8381 & 0.8134 \\
\hline SVM & $\mathrm{CW}$ & $\begin{array}{c}\text { C: '10', } \\
\text { class_weight: 'balanced', } \\
\text { gamma: '0.0001', } \\
\text { kernel: 'rbf'' }\end{array}$ & 809 & 7 & 10 & 204 & 0.0467 & 0.5882 & 0.1773 & 0.7951 & 0.6934 & 0.7986 & 0.7538 \\
\hline
\end{tabular}

Tabela II. Melhores modelos obtidos para cada técnica previamente definida.

Classificador: $\mathrm{DT}=$ árvore de decisão; $\mathrm{LR}=$ regressão logística; $\mathrm{SVM}=$ máquina de vetores de suporte.

Técnica: $\mathrm{ROS}=$ random over sampling; $\mathrm{RUS}=$ random under sampling; $\mathrm{CW}=$ class weighting. Fonte: Dados da pesquisa.

Para o modelo de melhor performance, o de regressão logística, em relação ao classificador de referência, foi possível alcançar um incremento de aproximadamente $300 \%$ à revocação, $37,7 \%$ à medida $F_{2}, 32,7 \%$ à acurácia balanceada e $17,8 \%$ à área sob a curva ROC.

\section{INTERPRETABILIDADE DOS MÉTODOS}

Após a obtenção dos modelos verificou-se a adequabilidade da explicação alcançada para cada classificador.

\subsection{Regressão Logística}

Para a regressão logística, um método que resulta em um modelo caixa-branca, a relação entre as entradas e as saídas são distinguíveis a partir de seus coeficientes. Usando o pacote "Eli5" ("Explain Like I'm 5") para visualizar como o modelo funciona, vemos na figura 1, à esquerda, seus coeficientes

\footnotetext{
${ }^{10}$ Mostra o quão frequente o classificador detecta instâncias pertencentes à classe minoritária: $V P \div(V P+F N)$

${ }^{11}$ Mostra o quão frequente o classificador está correto ao predizer uma instância da classe minoritária: $V P \div(V P+F P)$

${ }^{12}$ De subamostragem com Random Under Sampling e Cluster Centroids, de sobreamostragem com Random Over Sampling e Synthetic Minority Over-sampling Technique (SMOTE) e combinada com SMOTEEN, usando o pacote "imblearn", e de ponderação de classes diretamente no classificador ("class_weight").

${ }^{13} F_{2}$, acurácia, acurácia balanceada, especificidade e área sob a curva ROC.
} 
(incluindo o intercepto, "BIAS"), com destaque para o atributo DEV_ALINEAS_11E12_FLAG, que aparenta ser o atributo mais importante para o classificador; à direita, ao selecionar um exemplo "mau pagador" ( $\mathrm{y}=1)$, identificamos a contribuição de cada atributo (para modelos lineares, a contribuição é igual ao peso $\times$ valor do atributo) para a pontuação final ou probabilidade:

Fig. 1. Esquerda: Coeficientes do modelo. Direita: Exemplo predito como mau pagador.

\begin{tabular}{|cl}
\hline Weight? & Feature \\
+0.994 & DEV_ALINEAS_11E12_FLAG \\
+0.854 & LIQUIDADOS_DISC \\
+0.835 & NUMERODEPENDENTES \\
+0.751 & NRO_MAX_MESES_AD \\
+0.687 & PERC_MAX_CHQ_ESP \\
+0.420 & CC_ATIVO_FLAG \\
+0.389 & PATRIMONIO \\
+0.210 & ESCOLARIDADE \\
+0.199 & ESTADOCIVIL \\
+0.127 & PRODUTO \\
+0.110 & SALDO_MAX_VENCIDOS \\
+0.084 & SALDO_MD_VENCIDOS_12M \\
+0.058 & RENDA \\
-0.004 & NRO_MAX_MESES_ACIMA_60 \\
-0.035 & SUBREGIAO \\
-0.060 & NRO_MAX_DIAS_AD \\
-0.090 & REPACTUOU_FLAG \\
-0.322 & IDADE \\
-0.364 & PA \\
-0.593 & SALDO_MD_12M \\
-0.647 & MUNICIPIO \\
-0.762 & IAP_DISC \\
-0.857 & PERC_MD_CHQ_ESP_12M \\
-0.931 & <BIAS>
\end{tabular}

\begin{tabular}{|c|c|c|}
\hline Contribution? & Feature & Value \\
\hline+1.395 & NUMERODEPENDENTES & 1.671 \\
\hline+0.807 & MUNICIPIO & -1.247 \\
\hline+0.702 & PERC_MAX_CHQ_ESP & 1.022 \\
\hline+0.485 & IDADE & -1.506 \\
\hline+0.462 & LIQUIDADOS_DISC & 0.541 \\
\hline+0.329 & ESCOLARIDADE & 1.566 \\
\hline+0.293 & PA & -0.805 \\
\hline+0.268 & PRODUTO & 2.118 \\
\hline+0.220 & SALDO_MD_12M & -0.372 \\
\hline+0.132 & IAP_DISC & -0.173 \\
\hline+0.011 & NRO_MAX_DIAS_AD & -0.181 \\
\hline+0.000 & NRO_MAX_MESES_ACIMA_60 & -0.064 \\
\hline-0.001 & CC_ATIVO_FLAG & -0.001 \\
\hline-0.007 & SALDO_MAX_VENCIDOS & -0.065 \\
\hline-0.010 & SALDO_MD_VENCIDOS_12M & -0.122 \\
\hline-0.028 & RENDA & -0.487 \\
\hline-0.037 & PERC_MD_CHQ_ESP_12M & 0.043 \\
\hline-0.052 & REPACTUOU_FLAG & 0.580 \\
\hline-0.053 & SUBREGIAO & 1.505 \\
\hline-0.070 & ESTADOCIVIL & -0.351 \\
\hline-0.152 & PATRIMONIO & -0.391 \\
\hline-0.355 & NRO_MAX_MESES_AD & -0.473 \\
\hline-0.369 & DEV_ALINEAS_11E12_FLAG & -0.371 \\
\hline-0.931 & $\langle B \mid A S\rangle$ & 1.000 \\
\hline
\end{tabular}

Fonte: Dados da pesquisa.

4.2 Árvore de decisão

Para a árvore de decisão, um método que também resulta em um modelo caixa-branca, é possível verificar após ajuste do modelo (figura 2) a importância relativa de cada atributo ao fazer uma previsão, a partir da propriedade "feature_importances_" do classificador:

Fig. 2. Árvore de decisão: atributos mais relevantes para o classificador.

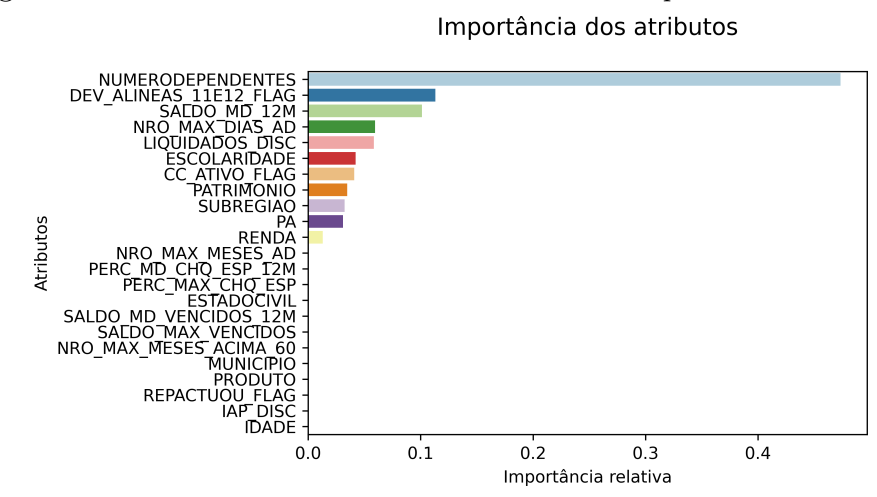

Fonte: Dados da pesquisa.

Adicionalmente, é também possível percorrer os ramos da árvore e verificar seus testes condicionais, permitindo explicar o modo como foi realizada a predição, conforme exibido na figura 3 : 
Fig. 3. Árvore de decisão: nós com testes condicionais.

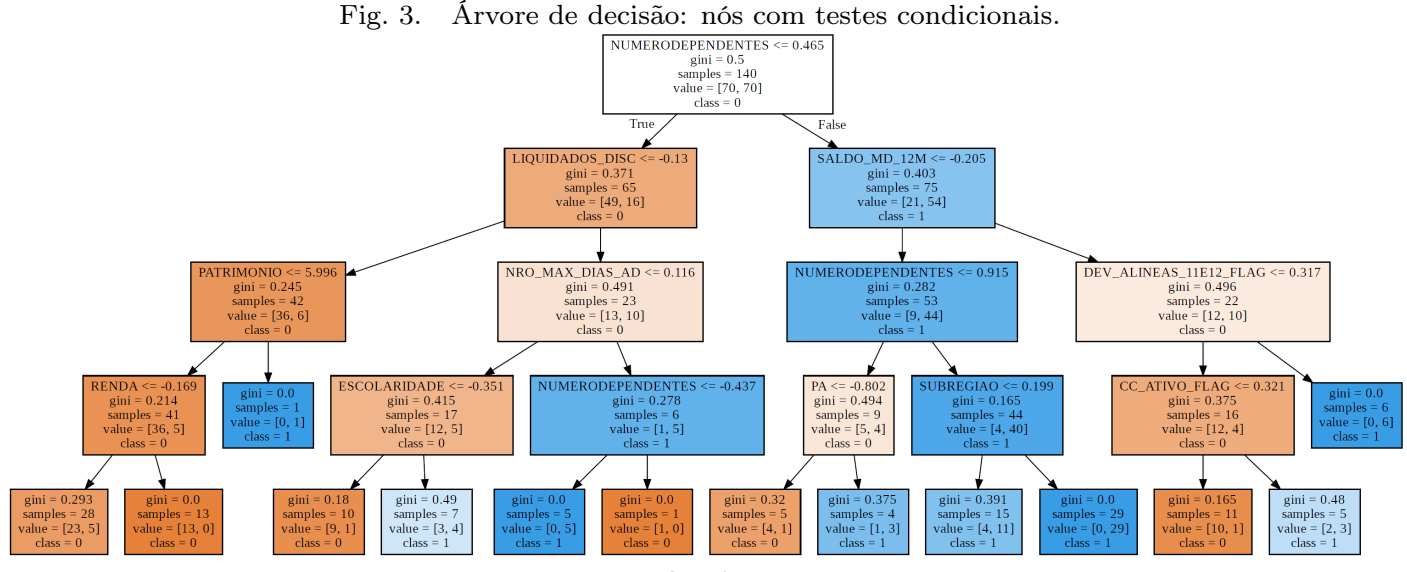

Fonte: Dados da pesquisa.

4.3 Máquina de vetores de suporte (Support Vector Machine (SVM))

Em relação à máquina de vetores de suporte, um método que resulta em um modelo caixa-preta, não é possível identificar como o modelo determina as suas saídas. Portanto, para interpretá-lo usaremos a SHAP, instanciando um "explicador" genérico (model-agnostic) a fim de explicar as predições do modelo a partir dos valores SHAP aproximados ${ }^{14}$, conquanto suficientes para racionalizar a decisão.

A seguir, na figura 4, uma decisão de crédito predita pelo classificador como "mau pagador":

Fig. 4. Máquina de vetores de suporte: visualização gráfica da contribuição de cada atributo à saída do modelo.

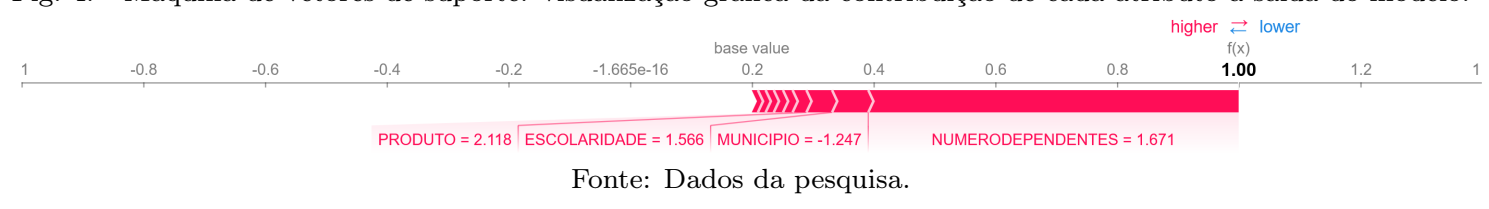

— $f(x)$ corresponde à predição - "1" para mau pagador;

- valor base (base value) representa o valor médio predito para o conjunto de dados, equivalente ao valor obtido se não conhecêssemos qualquer atributo à saída em análise;

- os atributos que aumentam a predição média são exibidos em vermelho, com o tamanho da faixa representando a magnitude do efeito do atributo, enquanto aqueles que reduzem o valor predito são exibidos em azul (não presentes neste exemplo na figura 4):

- o atributo "NUMERODEPENDENTES" teve grande impacto à classificação, sendo o valor absoluto observado para o exemplo $(-1.671)$ maior que o valor médio absoluto observado para o atributo (-0.547), referência à obtenção da contribuição marginal, o que "empurrou" a predição em direção a "1";

- o mesmo ocorreu, por exemplo, com os atributos "MUNICIPIO", "ESCOLARIDADE" e "PRODUTO".

Tomando o mesmo exemplo da figura 4, os valores $\mathrm{SHAP}^{15}$ antes representados graficamente pelas faixas em vermelho podem agora ser identificados individualmente junto aos seus valores aproximados, indicando o quanto cada atributo contribuiu para a predição "mau pagador" realizada pelo modelo caixa-preta (figura 5):

\footnotetext{
${ }^{14}$ Não há um "explicador" otimizado para máquinas de vetores de suporte.

${ }^{15}$ Contribuição marginal média ponderada de um atributo qualquer ao valor de saída do modelo.
} 
Fig. 5. Máquina de vetores de suporte: visualização gráfica alternativa da contribuição de cada atributo à saída do modelo.

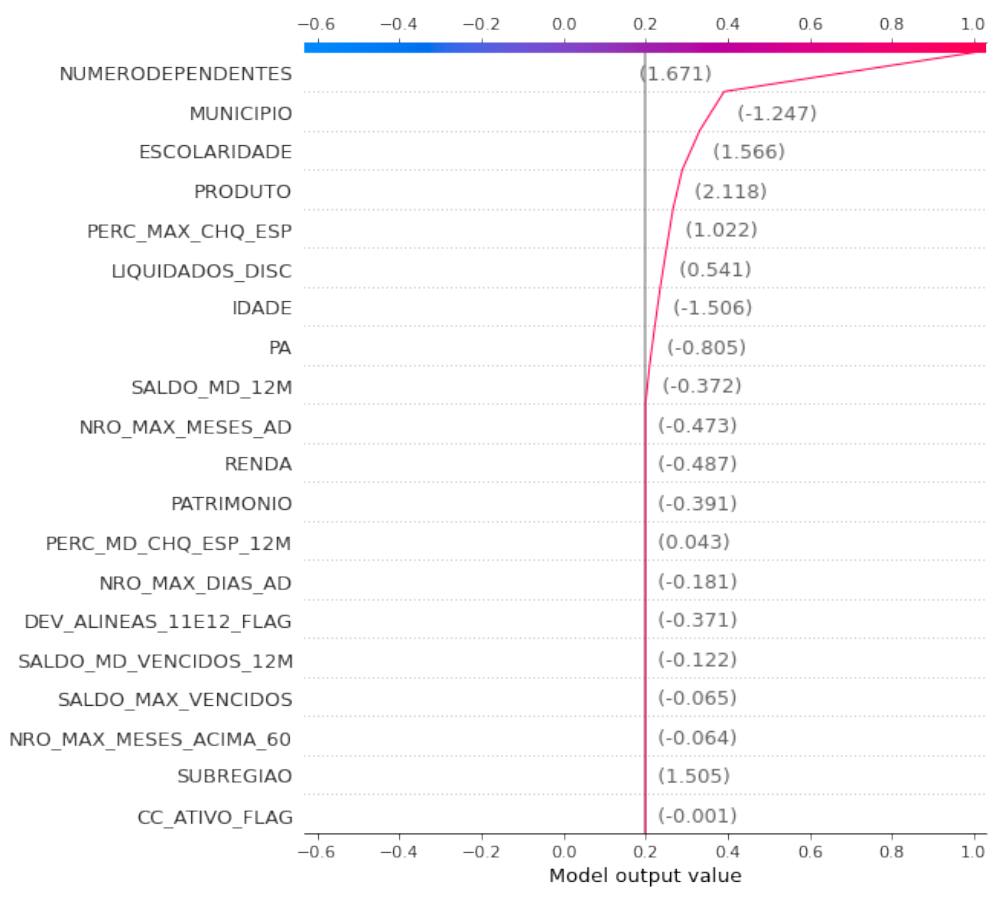

Fonte: Dados da pesquisa.

\section{CONCLUSÃO}

Sob a perspectiva da precisão, dentre os métodos escolhidos, a regressão logística apresentou o melhor desempenho. Por se tratar de um método caixa-branca, sua interpretação é aparente e não se requer qualquer modificação ao processo habitual de modelagem de credit scoring, diferentemente de quando empregados métodos caixas-pretas. Testemunhou-se que a melhor solução pode residir em uma abordagem simples, todavia eficaz.

À avaliação da interpretabilidade, foco da análise experimental, através da implementação de um método de interpretação local foi possível compreender e explicar as predições do método caixa-preta, ao se identificar os atributos de maior relevância para a predição, juntamente com a estimação de sua contribuição, revelando como cada atributo afetou a predição realizada e justificando adequadamente, e com precisão, a lógica do processo de tratamento de dados.

Logo, ao empregar métodos que produzam modelos caixas-pretas, sugere-se agregar ao processo habitual de modelagem de credit scoring a explicação/valor de Shapley, como exibido na figura 6:

Fig. 6. Etapa final do processo de modelagem de credit scoring acrescida da explicação/valor de Shapley.

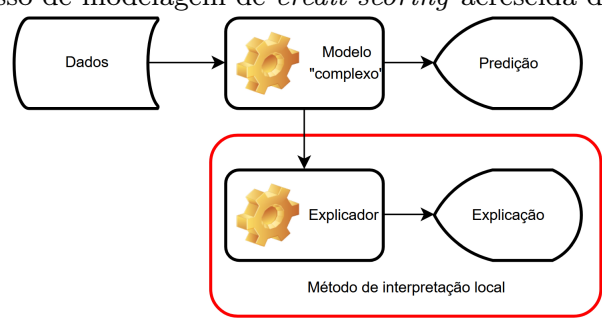

Fonte: Elaborada pelo autor. 
Ainda que a LGPD não explicite o que poderia ser considerada uma resposta aceitável ao titular dos dados, o Comitê Europeu para a Proteção de Dados (European Data Protection Board) um órgão consultivo independente para proteção de dados e privacidade, recomendou esclarecer [EDPB 2018]:

—as categorias de dados utilizadas à formação do perfil comportamental;

-uma síntese do porquê essas categorias são consideradas apropriadas;

- a razão pela qual esse perfil comportamental é relevante ao processo de decisão automatizado; e

- como esse perfil é utilizado para uma decisão relativa ao titular dos dados.

Para além disso, visando prover maior transparência ao tratamento de dados, pode-se apresentar quais dados especificamente foram utilizados, de onde vêm, quais os parâmetros de tratamento, tornando clara e compreensível a sua política e, com o esclarecimento oferecido pela SHAP, como esses dados contribuíram efetivamente à tomada de decisão. Tais explicações serão um dos principais instrumentos de apelação contra o tratamento automático realizado de forma inadequada.

Trabalhos futuros incluem a implementação de ensembles e de redes neurais profundas, e a variação da abordagem, desta vez sob a perspectiva de um problema de classificação de uma classe (OneClass Classification) - em que se assume como premissa que todo o conjunto de dados conteria apenas instâncias "normais" e que o público pertencente à atual classe positiva representaria instâncias anômalas - e também como uma tarefa de aprendizado incremental (online learning/data stream learning) - capaz de alterar dinamicamente sua estrutura sempre que surgirem novos dados - pois é esperado que cenários de estresse financeiro sejam cada vez mais recorrentes, o que exigirá rápida adaptação à nova realidade.

\section{REFERENCES}

Batista, M. R. S. A utilização de algoritmos de aprendizado de máquina em problemas de classificação. Universidade de São Paulo (USP), 2018.

EDPB. EUROPEAN DATA PROTECTION BOARD. Article 29 Data Protection Working Party. Guidelines on automated individual decision-making and profiling for the purposes of regulation 2016/679. EDPB, Feb., 2018.

Erickson, A. Comparative analysis of the EU's GDPR and Brazil's LGPD: Enforcement challenges with the LGPD. Brooklyn Journal of International Law 44 (2): 859-888, 2019.

Hand, D. J. And Henley, W. E. Statistical classification methods in consumer credit scoring: A review. Journal of the Royal Statistical Society. Series A (Statistics in Society) 160 (3): 523-541, 1997.

Louzada, F., Ara, A., And Fernandes, G. B. Classification methods applied to credit scoring: Systematic review and overall comparison. Surveys in Operations Research and Management Science 21 (2): 117-134, 2016.

Molnar, C. Interpretable machine learning, 2020.

Oliveira-Junior, R. A. A. Um estudo acerca dos métodos estatísticos clássicos e de inteligência artificial aplicados ao desenvolvimento de credit scoring à luz da nova Lei Geral de Proteção de Dados brasileira. Universidade de São Paulo (USP), 2021.

Ribeiro, M. T., Singh, S., And Guestrin, C. "Why should I trust you?": Explaining the predictions of any classifier. In Proceedings of the 22nd ACM SIGKDD International Conference on Knowledge Discovery and Data Mining. KDD '16. Association for Computing Machinery, New York, pp. 1135-1144, 2016.

Santo, J. L. C. F. A semiotic view on the interpretability of machine learning models. Pontifícia Universidade Católica do Rio de Janeiro (PUC-Rio), 2018.

Thomas, L. C., Crook, J. N., And Edelman, D. B. Credit scoring and its applications. Society for Industrial and Applied Mathematics, New York, 2002. 